\title{
Imaging without contrast to improve safety in patients with impaired renal function
}

A new study has demonstrated the feasibility of measuring total renal volume without the need for contrast medium.

In patients with autosomal dominant polycystic kidney disease (ADPKD), total renal volume is a measure of disease progression that is commonly assessed by CT and MRI. However, a disadvantage of these techniques is that they often use contrast medium, which can be nephrotoxic, especially in patients with impaired renal function. Also, the kidney contours on images have to be manually traced, which is a labor-intensive and subjective process. Therefore, Renzo Mignani and colleagues developed a semi-automated method utilizing axial $\mathrm{T}_{2}$-weighted MRI without contrast medium, which was tested for its accuracy in a cohort of patients with ADPKD.

Initially, a phantom was constructed to simulate a kidney with cysts. To calculate total renal volume, a point was selected by

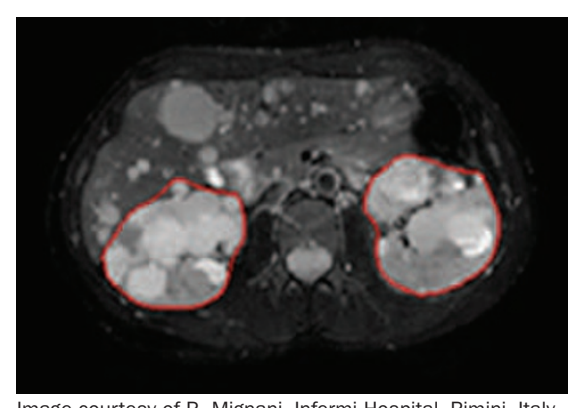

Image courtesy of R. Mignani, Infermi Hospital, Rimini, Italy.

the operator within a slice of each kidney and contours were automatically extracted. The calculated volume was compared with values obtained through stereology, which takes about 20 min per kidney. The error for the automated procedure was $-1.9 \%$ when compared with the true phantom volume. To test the technique, a total of 30 patients with ADPKD, of whom 22 had normal renal function and eight had chronic kidney disease, underwent MRI. The mean error for kidney volume assessment using the automated method was $-5 \pm 37 \mathrm{ml}$ in absolute terms, and $-0.6 \pm 9.6 \%$ in percentage terms. No difference was found between observers.

Although the automated method could calculate total renal volume in $3 \mathrm{~min}$, MRI acquisition takes about $12 \mathrm{~min}$. In addition, variability between MRI scanners means that calibration of the technique for different scanners is likely to be necessary. "The results of this study warrant further research geared towards automatic quantification of cyst volume and disease progression in a larger group of patients with ADPKD and chronic kidney disease," concludes Mignani.

Helene Myrvang

Original article Mignani, R. et al. Assessment of kidney volume in polycystic kidney disease using magnetic resonance imaging without contrast medium. Am. J. Nephrol. 33, 176-184 (2011) 